\title{
The role of extracorporeal shock wave lithotripsy in renal calculi
}

\author{
Neville D Perera, Sohan JS Perera, \\ Department of Urology, The National Hospital of Sri Lanka
}

\section{Key words: Shock wave lithotripsy, renal calculi. \\ Introduction}

Extracorporeal shock wave lithotripsy (ESWL/SWL) exemplifies one of the most innovative and revolutionary non-invasive techniques in treating urinary lithiasis. Following initial identification of the effects of shock waves on hard objects by Russians in 1950s without reproducible results, first successful animal studies were reported in 1971.The first medically applicable lithotripter was produced by Dornier a German air craft manufacturer. The concept was developed in West Germany in the early1980s which gained popularity due to its noninvasiveness, high efficacy in treating majority of kidney and ureteral stones. The first successful human treatment was reported in Munich Germany, February 1980, using the Human Model 1(HM1) which comprised of a large water tank in which the anaesthetized patient was submerged [1](fig. 1) and was bulky in comparison to modern compact machines which fits in to a reasonably sized outpatient clinic room or a space of a caravan in case of the mobile lithotripters(fig. 2). Initially the use was limited by the non availability, especially in the developing world, due to high initial and maintenance cost, but gained wide acceptance as the first line of treatment for renal stones. The first lithotripter in Sri Lanka was installed at the National Hospital of Sri Lanka in 1992 and since then approximately 25003000 procedures are done per year at this centre.

Correspondence :

Perera ND

Email : nevilledperera@yahoo.com

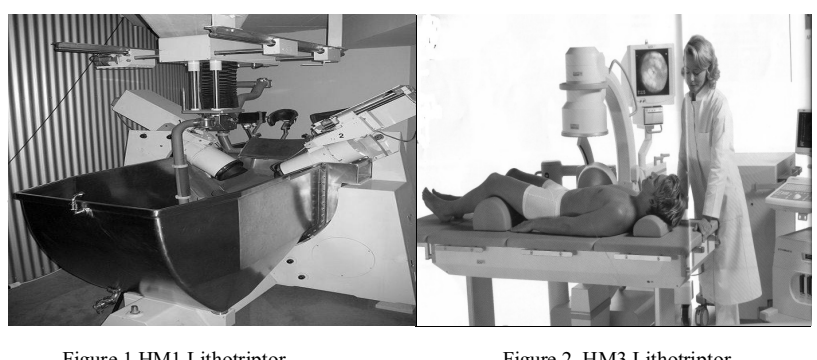

Figure 1.HM1 Lithotriptor

Figure 2. HM3 Lithotriptor

\section{Mechanism of stone disintegration}

The shockwaves travel across soft tissue with minimal release of energy and tissue injury and when it encounters a high density object (e.g. stone, bone), releases the energy at the stone-tissue interface similar to the effects of a high velocity bullet traveling through the body. The shock wave causes compression, shattering and erosion (at entry and exit sites) of the stone leading to fragmentation. Of these various forces, the generation of compressive and tensile forces and cavitation are thought to be the most important. In cavitation, shockwave energy applied at a focal point leads to failure of the liquid with generation of water-vapor bubbles. These gaseous bubbles collapse explosively, creating microjets that fracture and erode the calculus. The destructive forces generated when the cavitation bubbles collapse are responsible for the ultimate stone fragmentation. Repeated waves lead to pulverization of the calculi into small fragments (ideally $<1 \mathrm{~mm}$ ) small enough to travel down the ureter for spontaneous expulsion.

However repeated application of waves can also cause some degree of trauma to thin-walled vessels in the kidneys and adjacent tissues which result in haemorrhage, release of cytokines/and possible chronic loss of tissue function, which is well proven in canine and human studies [2].

\section{Procedure}

In modern lithotripters patient is positioned on the machine and the calculus is focused using $\mathrm{X}$ rays or ultrasound. The lithotripter is within a water balloon which is kept on the patient so that the calculus lies 
within the focal zone of the lithotripter. It is generally done as a day procedure and patient is observed for 6 hours for colics, haematuria, internal bleeding and fever with sepsis and discharged on analgesics. A short course of antibiotics is recommended in case the patient has recently recovered from urinary sepsis or is immuno-compromised. There are no clear guidelines for the use of post procedure antibiotics in diabetics and patients with urinary tract anomalies eg reflux disease, PUJ obstruction or horse shoe kidneys. Requirement of analgesia is important in achieving successful fragmentation as patient movement could defocus the shock wave. Non steroidal anti inflammatory drugs in the parenteral form (intra rectal one hour before) with or without opioids is the commonest combination. However more sensitive patients would need sedation or even a short anesthetic. Strong analgesia is required when higher intensity of energy is used for a hard stone over a prolonged period. ESWL in children is done under general anaesthesia for best results [3].

\section{The shock wave machines}

All lithotripsy machines have 4 main components (fig.3)A shockwave generator, (2) a focusing system, (3) a coupling mechanism, and (4) an imaging/localization unit.

\section{Shockwave generator}

Shockwaves can be generated in 1 of 3 ways

- Electrohydraulic-: Used in first human model (HM1) where a high-voltage electrical current passes across a spark-gap electrode located within a water-filled container. The discharge of energy produces a vaporization bubble, which expands and immediately collapses, thus generating a high-energy pressure wave.

- Piezoelectric: Produces electricity via application of mechanical stress. Piezoelectric ceramics or crystals, are stimulated by high-frequency electrical pulses. The alternating stress/strain changes create ultrasonic vibrations, resulting in a shockwave.

- Electromagnetic: The most modern method which applies a high voltage to an electromagnetic coil, (similar to the effect in a loudspeaker). This induces high-frequency vibration to produce shockwaves. Most current lithotriptors are powered by an electromagnetic generator and their focusing units are capable of delivering shockwaves with smaller focal zone, minimizing damage to surrounding soft tissue[4]. However a smaller focal zone, causes the stone to move out of the target zone making newer generation machines less effective. This explains the higher success rates noted in children who undergo SWL under general anesthesia with better control of respiratory and body movements [5].

\section{Focusing systems}

The focusing system is used to direct the shockwaves at a focal point in a synchronous fashion. The basic geometric principle used in most lithotriptors is that of an ellipse. Shockwaves are created at one focal point (F1) and converge at the second focal point (F2). The Focusing systems differ, depending on the shockwave generator used. Electrohydraulic systems used the principle of the ellipse; and in electromagnetic systems, the shockwaves are focused with either an acoustic lens or a cylindrical reflector.

\section{Coupling mechanisms}

In the propagation and transmission of a wave, energy is lost at interfaces with differing densities. As such a coupling system is needed to minimize the dissipation of energy of a shockwave as it traverses the skin surface. The usual medium is water, as this has a density similar to that of soft tissue and is readily available. In first-generation lithotriptors (Dornier HM1), the patient was placed in a water bath. However, newer lithotripters use small water-filled drums or bubbles with a silicone membrane These modifications helps to pass the shock wave to the body without much turning of the patient and also the discomfort of getting soaked in a water bath[6] .

\section{Stone localization}

The imaging methods commonly used to localize stones include fluoroscopy and ultrasonography.

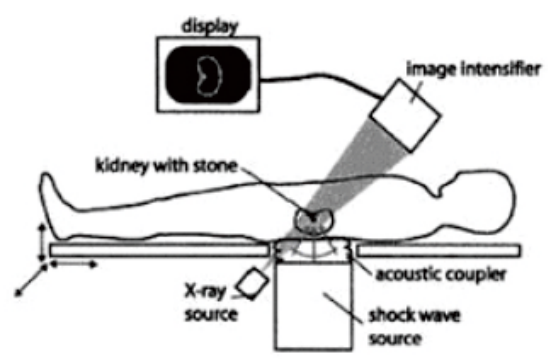

Figure 3 Parts of lithotriptor 
Fluoroscopy, though familiar, involves radiation but is excellent for detecting radio-opaque stones, both in the kidney and the ureter. Radiolucent stones (eg, uric acid stones) will need, intravenous contrast or on table retrograde pyelography

Ultrasonography visualizes both radiopaque and radiolucent renal stones in real-time, though limited by its operator dependency and failure to detect ureteral calculi due to interposed intestinal loops. The newest-generation lithotriptors often provide imaging with both fluoroscopy and ultrasonography. The ability to alternate between imaging modalities allows the urologist to compensate for the deficiencies of either system.

\section{Indications for SWL}

The American Urological Association Stone Guidelines Panel has classified ESWL as a potential first-line treatment for ureteral and renal stones smaller than $2 \mathrm{~cm}$. Its place in the management of mid and lower ureter is controversial .While larger mid ureteric stones $(1-2 \mathrm{~cm})$ responds to SWL, success rate in localizing smaller stones is not satisfactory. For proximal ureteral stones there has been a higher success rate $(65 \%-81 \%)$ than for lower ureteral stones $(58 \%-67 \%)$. ESWL is less effective as an initial treatment in comparison to ureteroscopic lithotripsy. It is indicated for all ages except in infancy where it could pose technical difficulties in focusing system.

\section{Contraindications}

Absolute contraindications to extracorporeal shockwave lithotripsy (ESWL) include:

- Acute urinary tract infection or ongoing urosepsis which could lead to even fatal septecamia and renal rupture.

- Uncorrected bleeding disorders or coagulopathies could lead to massive haemorrhage.

- Pregnancy, as the effect on the fetus and placenta is not clearly understood.

- Distal obstruction where fragments would cause further obstruction and infection.

Relative contraindications include:

- Abnormal body habitus: Morbid obesity and severe kyphoscoliois or spinal deformities (ankylosing spondilitis) may prevent proper positioning. It is shown that skin to stone distance has an inverse relationship with the success in stone fragmentation.

- Adverse calyceal anatomy with poor intrarenal drainage (eg, infundibular stenosis, dependant calyces with narrow long necks) leads to poor stone clearance and higher residual fragments.

- Kidney malformations (eg. horseshoe, crossed ectopic and pelvic kidneys) give poor out comes

- Poorly controlled hypertension associated with a higher chance of haematuria and haematoma formation.

- Renal insufficiency: As SWL could lead to micro haemorrhages and paranchymal fibrosis in the long run, repeated ESWL is best avoided in kidneys with chronic parenchymal injury. It is also shown that stone-free rates in patients with renal insufficiency (57\%) (serum creatinine level of $2-2.9 \mathrm{mg} / \mathrm{dL}$ ) were significantly lower than in patients with better renal function $(66 \%)$ (serum creatinine level $<$ $2 \mathrm{mg} / \mathrm{dL}$ ).

- Rarely some gastrointestinal disorders (eg.ulcerative colitis ) could be exacerbated after ESWL treatment.

\section{Limiting factors of ESWL \\ Density of the stone}

Stones which are too hard with high Hounsfield numbers in the CT scan (more than 1000 ) or too soft calcified matrix stones and renal papillary stones are not suitable for SWL. Thus fragmentation rate of cystine and calcium oxalate monohydrate stones is low[7].

\section{Stone size}

Too large $(>2 \mathrm{~cm})$ stones will leave several residual fragments and would require several sessions with added complications. However it can be used in combination with percutaneous nephrolithotomy(PCNL), for residual stone after debulking the stone with latter. Too small $<0.5 \mathrm{~cm}$ calculi will cause difficulties in on-table localization with a high failure rate. The ESWL success rate decreases as the stone size increases. Chaussy et al, who are pioneers in SWL, in 1984 reported a stone free rate of $91 \%$ for stones less than $2 \mathrm{~cm}$ stones and $50 \%-70 \%$ for stones $2-3 \mathrm{~cm}$ and dramatically decrease further for staghorn stones [8][9].

\section{Calyceal anatomy}

This is one of the main factors overlooked by many 
referring clinicians. It is best studied in a three film IVU or a CT IVU and a calculus in a closed calyx or a lower calyx with a long narrow vertical neck (ureterocalyceal angle $<30^{\circ}$ degree) are associated with poor results[10]. Stone-free rates are $29 \%$ for patients with lower pole calculi of $11-20 \mathrm{~mm}$ and $20 \%$ for those with calculi $>20 \mathrm{~mm}$. The presence of multiple stones has also been related to higher residual stones and failure after SWL [11].

Shock wave rate affects stone fragmentation in vitro and in vivo with improved ESWL efficiency occurring at slower rates similarly, progressive increase in lithotripter output voltage improves stone fragmentation in vitro. However, only two clinical studies have addressed the effect of varying shock wave rate on the efficiency of stone fragmentation [12].

\section{Tissue effects and complications of ESWL Incomplete fragmentation}

Although the desired goal is total pulverization with complete clearance, incomplete fragmentation with large residual stone fragments can lead to steinstrasse (German) (uretric "stone street") which usually cause minimal obstruction(fig. 4), or obstruction by larger fragments leading to obstructed and even non functioning renal unit. When left alone, growth of residual renal fragments over $4 \mathrm{~mm}$ could occur in $21 \%-59 \%$ of patients with $43 \%$ risk of needing an intervention, within 2 vears with residual calculi.

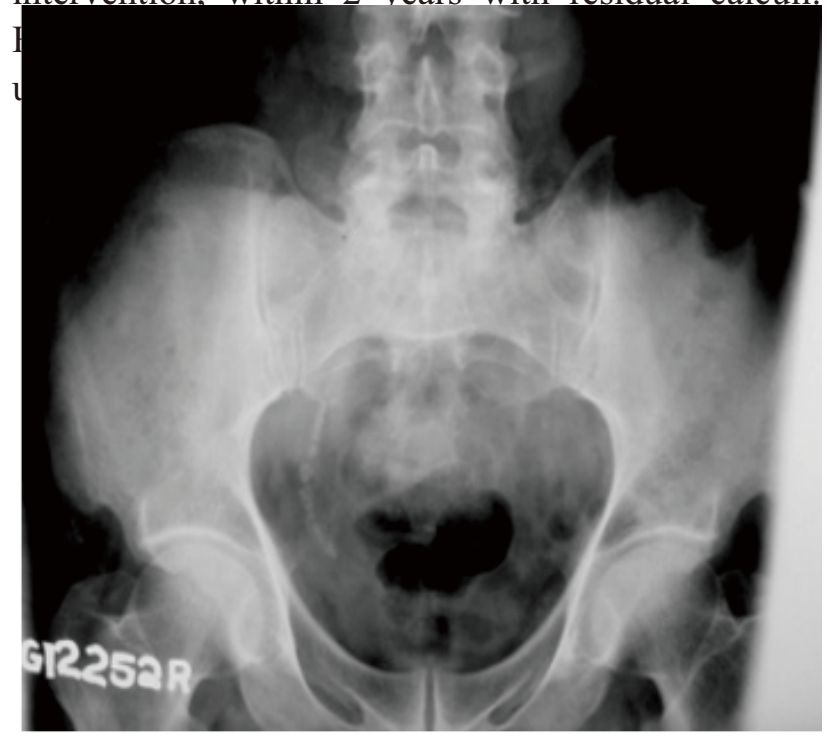

Figure. 4 Steinstrasse following SWL
$\mathrm{X}$ ray should be taken in 6 weeks after SWL. Fragments will pass by that time and minor cracks in the calculus will be visible after 6 weeks. Most studies have shown that particles pass in 10-13 days. There is strong evidence to show that the alfa adrenergic blocker tamsulosin after SWL increases the stone free rate (medical expulsion therapy) [14].

Place of pre procedure ureteral stenting is well established in SWL.When dealing with larger stones $(1.5$ to $2 \mathrm{~cm})$ studies have shown that there is a significant advantage in double J stenting before SWL compared to in situ therapy, in terms of avoidance of steinstrase. Some studies have shown increase fragmentation in the presence of the stent which creates an additional inter phase for the shock wave to pass through. However it does not benefit stone free rates and in fact could delay the passage of fragment due to paralysis of the peristalsis. Hence it is advisable to remove the stent as soon as the fragmentation is completed[15].

\section{Post SWLinfection and sepsis}

The local mucosal injury and release of bacteria from stone can cause bacteriuria, bacteraemia, urosepsis, perinephric abscess, endocarditis, candida and Klebsiella endophthalmitis, candida septicaemia, tuberculosis, and even death after ESWL[16]. Bacteriuria has been found in $7.7 \%-23.5 \%$ even without evidence of any infection at the time of the procedure.

Symptomatic UTI occurs more in patients with infective (triple phosphate), multiple or complex stones and with pre SWL urologic manipulations. Although bacteraemia incidence is around 14\% fortunately only $<1 \%$ of cases end up in overt sepsis. Increased risk is noted when urine culture is positive or in the presence of obstruction [17]. C-reactive protein (CRP) measurement on the first and third days after ESWL is useful for early diagnosis as it increases, before positive urine and blood cultures. Indications for early antibiotics with empirical therapy based on CRP remains unclear[18].

The role of routine prophylactic antibiotics is also controversial. A meta-analysis indicates routine prophylactic antibiotics in all patients who undergo ESWL which is efficacious and cost effective in decreasing the need for inpatient treatment of urosepsis. However, several other studies, including randomized controlled trials, have demonstrated no 
advantage of prophylactic antibiotics in patients without preoperative UTI or infection. Hence preoperative antibiotics should be reserved to patients with infection-related stones (staghorn and struvite calculi), positive urine cultures, or a history of recurrent UTIs and to those who undergo instrumentation at the time of ESWL[19].

\section{Renal complications}

Histopathological examination of human and canine kidneys showed endothelial cell damage to midsized arteries, veins, and glomerular capillaries immediately after ESWL [20]. Thin-walled arcuate veins rupture easily leading to haematuria and haematoma. Fortunately as it is a focal effect most of the renal parenchyma is unaffected unless longer duration and repeated attempts have taken place.

Severe injury to the nephron, microvasculature, and the surrounding interstitium renal tubules and vessels has been reported. These injuries may be related to the long-term effects such as fibrosis and hypertension [21]. Microscopic and occasionally gross haematuria usually resolves spontaneously in a few days. Intrarenal, subcapsular, or perirenal fluid collections occur in $<1 \%$ of patients who undergo ESWL. If actively investigated with $\mathrm{CT}$ scan this could rise to $20 \%-25 \%$. Haemorrhage and haematomas may be related to the type of lithotripter used. The HM1, which uses electrohydraulic energy, delivers more energy per shock wave into the kidney and causes more trauma than the newer electromagnetic source. Antiplatelet drugs, hypertension, obesity, diabetes mellitus, and the number and intensity of shockwaves are risk factors which increase the renal injury [22].

Treatment of the haematomas is mainly conservative with spontaneous resolution within two years without clinically evident adverse effects on blood pressure or renal function [23](figure 5)This patient developed a haematoma following SWL which was managed concervatively. However a patient with acute renal failure and hypertension or a solitary kidney could develop renal impairment [24].

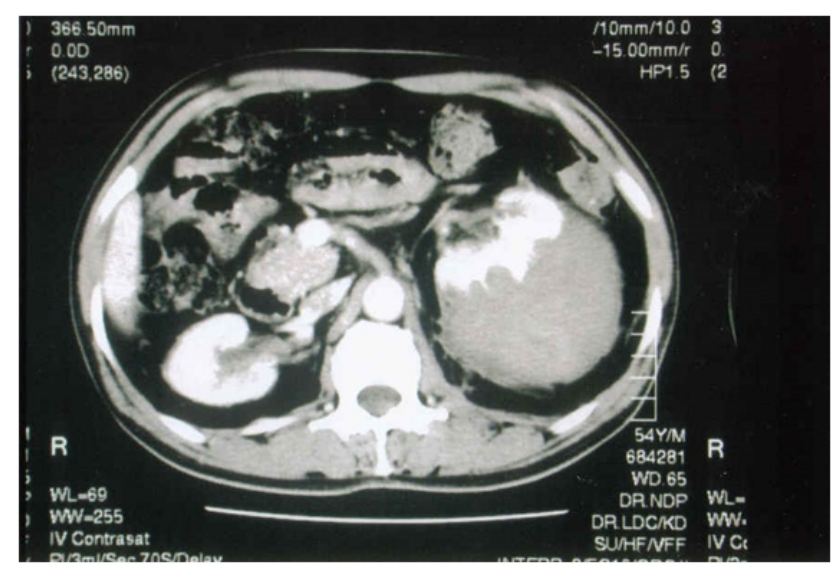

Figure 5 left renal haematoma following SWL

\section{Renal function after ESWL}

There is biochemical evidence of renal injury with ESWL with a rise of indicators such as renin, creatinine, N-Acetyl-b-D-glucosaminidase(NAG), galactosidase, -2-microglobulin, and proteinuria rise which return to near-normal levels within a few days[25]. Reduction of glomerular filtration rate (GFR) and renal plasma flow occur transiently and does not affect GFR over the long-term. Controversies prevail on the detrimental effect of shock waves on children's growing kidneys [26]. Shock wave energy induces transient functional damage of tubular function in children. Significant alterations of renal growth in children after ESWL were observed in one long-term study, but assessment of long-term effects of ESWL on GFR in children by renal isotope studies did not show any parenchymal damage in children who underwent ESWL[27]. Minimizing the power and number of shocks may decrease the renal damage. Increasing evidence suggests that slower shock wave, although resulting in better fragmentation, could cause more renal damage [28].

\section{ESWL and hypertension}

Studies indicated that renal stone disease by itself significantly increases blood pressure irrespective of the type of treatment; the exact impact of SWL on hypertension is still unclear. Randomized controlled trials failed to prove that ESWL causes changes in blood pressure [29][30]. The incidence of newly diagnosed hypertension in those who have undergone ESWL, does not differ from the incidence within the general population, which is approximately $6 \%$. Increase of diastolic hypertension alone, has been found in multiple studies, which increased with the number of shock waves. Patients over the age of 60 
may develop hypertension at a higher rate than the control population, which can be predicted by increased intra renal resistive indices. Close postoperative screening for hypertension in the older patients is recommended [31].

\section{Cardiovascular complications during ESWL}

It is worth noting that preexisting pulmonary and cardiac problems are not contraindications to ESWL, provided they are under control. While minor cardiac arrhythmias are not uncommon with an incidence of $11 \%-59 \%$, significant myocardial events are extremely rare. With a history of cardiac arrhythmias, the shockwave can be linked to electrocardiography (ECG), thus firing only on the $\mathrm{R}$ wave in the cardiac cycle, coinciding with the refractory period of the cardiac cycle (named "gated lithotripsy"). Commonly seen ESWL-induced ventricular ectopics are probably reflective of mechanical stimulation of the myocardium rather than myocardial injury. Based on studies, patients with preexisting cardiac disease, without documented preoperative arrhythmias, can probably undergo ungated lithotripsy safely [32].

No correlation is demonstrated between post ESWL arrhythmias and premature beats, age, gender, heart disease, stone size, ureteral stents or nephrostomy tube, mode of anaesthesia, number of shock waves, or types of lithotriptors.

ESWL is safe with pacemakers when done under guidance of the cardiologist. Dual-chamber pacemakers should be reprogrammed to the singlechamber mode, and single-chamber rate-responsive devices should have the activity mode programmed off. Patients with pacemakers in the abdomen should avoid the direct impact of the shocks [33].

In spite of few reported abdominal aortic aneurismal ruptures after ESWL there is still evidence for safety of SWL with aneurysms. Inferior caval thrombosis is a very rare complication [34].

To avoid haemorrhagic complications oral anticoagulants (e.g. clopidogrel and warfarin, aspirin) and non-steroidal anti-inflammatory drugs (NSAIDs) are best avoided for 7 days before treatment. Hypertension and renal impairment are relative contraindications.

\section{Gastrointestinal injury secondary to ESWL}

Small bowel and colon perforation, ureterocolic fistula formation, gastrointestinal anastomotic dehiscence, caecal ulcers, colon erythema, bruising and haematomas, bleeding per rectum, pancreatitis and peripancreatic haematoma and abscesses, subcapsular haematomas of liver and spleen have been reported but are rare. As expected these were associated with increase in the number and level of energy of shock waves and prone position which is no longer necessary in newer machines with movable shock heads [35].

\section{Effects of ESWL on fertility and pregnancy}

There is no strong evidence to suggest permanent effects on testicular and ovarian function. Pregnancy is the only absolute contraindication to ESWL because of the potential harmful effects on the foetus found in experimental studies, such as spontaneous abortion and placental disruption. However, there are reports of successful healthy deliveries with no detectable congenital malformations or chromosomal anomalies, despite accidental application of ESWL during pregnancy [36].

\section{Conclusions}

ESWL is a convenient, safe method to treat medium sized stones especially in the upper urinary tract when proper indications are followed. Experience gained over 3 decades with better understanding of its tissue effects, and reported concerns (of which most are rare and do not hamper the effectiveness ESWL) SWL still remains as a strong and reliable, minimal access method in the armamentarium of the urologist. With the advancement of competitive techniques such as endoscopy and percutaneous renal surgery, it is mandatory to pay attention to details of the limitations of ESWL to minimize failure rates and complications.

\section{References}

1. Moody JA, Evans AP, Lingeman JE. Extracorporeal shockwave lithotripsy. In: Weiss RM, George NJR, O'Reilly PH, editors. Comprehensive Urology. Mosby International Limited;2001. p. 623-36.

2. Portis AJ, Yan Y, Pattaras JG, Andreoni C, Moore R, Clayman RV. Matched pair analysis of shock wave lithotripsy effectiveness for comparison of lithotriptors. J Urol 2003; 169:58-62.

3. Putman SS, Hamilton BD, Johnson DB. The use of shock wave lithotripsy for renal calculi. Curr Opin Urol 2004; 14:117-21.

4. Sheir KZ, Madbouly K, Elsobky E. Prospective randomized comparative study of the effectiveness and safety of electrohydraulic 
and electromagnetic extracorporeal shock wave lithotriptors. J Urol 2003;170:389-92.

5. Muslumanoglu AY, Tefekli A, Sarilar O, Binbay M, Altunrende F, Ozkuvanci U. Extracorporeal shock wave lithotripsyas first line treatment alternative for urinary tract stones in children: a large scale retrospective analysis.J Urol 2003;170:2405-8.

6. Paterson RF, Kuo RL, Lingeman JE. The effect of shock wave delivery on the efficiency of lithotripsy. Curr Opin Urol 2002;12:291-5.

7. Zhong P, Preminger GM. Mechanisms of differing stone fragility in extracorporeal shock wave lithotripsy. J Endourol 1994;8:263-8.

8. Chaussy C, Schuller J, Schmiedt E, Brandl H, Jocham D, Liedl B. Extracorporeal shockwave lithotripsy (ESWL) for treatment of urolithiasis. Urology 1984;23:59-66.

9. Wirth MP, Theiss M, Frohmuller HG. Primary extracorporeal shockwave lithotripsy of staghorn renal calculi. Urol Int 1992;48:71-5.

10. Lingeman JE. Prospective randomized trial of extracorporeal shock wave lithotripsy and percutaneous nephrostolithotomy for lower pole nephrolithiasis: initial long-term follow up. JEndourol 1997;11:95.

11. Abdel-Khalek M, Sheir KZ, Mokhtar AA, Eraky I, Kenawy M, Bazeed M. Prediction of success rate after extracorporeal shock-wave lithotripsy of renal stones- a multivariate analysis model. Scand J Urol Nephrol 2004;38:161-7.

12. Madbouly K, El-Tiraifi AM, Seida M, ElFaqih SR, Atassi R, Talic RF. Slow versus fast wave lithotripsy rate for urolithiasis: a prospective randomized study. J Urol 2005;173:127-30.

13. Sun BY, Lee YH, Jiaan BP, Chen KK, Chang LS, Chen KT. Recurrence rate and risk factors for urinary calculi after extracorporeal shock wave lithotripsy. J Urol 1996;156: 903-5, discussion, 906.

14. Streem SB, Yost A, Mascha E. Clinical implications of clinically insignificant store fragments after extracorporeal shock wave lithotripsy. J Urol 1996;155:1186-90.
15. Bierkens AF, Hendrikx AJ, Lemmens WA, Debruyne FM. Extracorporeal shock-wave lithotripsy for large renal calculi: the role of ureteral stents. A randomized trial. J Urol 1991;145:699-702.

16. Muller-Mattheis VG, Schmale D, Seewald $\mathrm{M}$, et al. Bacteriemia during extracorporeal shock wave lithotripsy of renal calculi. J Urol 1991;146:733-6.

17. Zink RA, Frohmueller HG, Eberhardt JE, et al. Urosepsis following ESWL. J Urol 1988;139:265A.

18. Yilmaz E, Batislam E, Tuglu D, Kilic D, Basar M, Ozluk O, Basar H. C-reactive protein in early detection of bacteriemia and bacteriuria after extracorporeal shock wave lithotripsy. Eur Urol 2003;43:270-4.

19. Pettersson B, Tiselius HG. Are prophylactic antibiotics necessary during extracorporeal shockwave lithotripsy? Br J Urol 1989;63:449-52.

20. Karlsen SJ, Smevik B, Hovig T. Acute morphological changes in canine kidneys after exposure to extracorporeal shock waves: A light and electron microscopic study. Urol Res 1991;19:105-15.

21. Delvecchio F, Auge BK, Munver R, Brown SA, Brizuela R, Zhong P, et al. Shock wave lithotripsy causes ipsilateral renal injury remote from the focal point: the role of regional vasoconstriction. J Urol 2003;169:1526-9.

22. Dhar NB, Thornton J, Karafa MT, Streem $\mathrm{SB}$. A multivariate analysis of risk factors associated with subcapsular hematoma formation following electromagnetic shock wave lithotripsy. J Urol 2004; 172:2271-4.

23. Krishnamurthi V, Streem SB. Long-term radiographic and functional outcome of extracorporeal shock wave lithotripsy induced perirenal hematomas. J Urol 1995;154:1673-5.

24. Williams CM, Kaude JV, Newman RC, Peterson JC, Thomas WC. Extracorporeal shock wave lithotripsy: long-term complications. AJR Am J Roentgenol 1998;150:311-5.

25. Momose A, Funyu T, Takahashi N, Suzuki T. Effect of pressure distribution of 
shockwave on renal hemorrhage after extracorporeal shockwave lithotripsy: comparison of EDAP LT-01 and Siemens Lithostar. J Endourol 1999;13:165-71.

26. Rutz-Danielczak A, Pupek-Musialik D, Raszeja-Wanic B. Effects of extracorporeal shock wave lithotripsy on renal function in patients with kidney stone disease. Nephron 1998;79:162-6.

27. Lifshitz DA, Lingeman JE, Zafar FS, Hollensbe DW, Nyhuis AW, Evan AP. Alterations in predicted growth rates of pediatric kidneys treated with extracorporeal shock-wave lithotripsy. J Endourol 1998;12:469-75.

28. Villanyi KK, Szekely JG, Farkas LM, Javor E, Pusztai C. Short-term changes in renal function after extracorporeal shock wave lithotripsy in children. J Urol 2001;166:222-4.

29. Jewett MA, Bombardier C, Logan AG, Psihramis KE, Wesley-James T, Mahoney JE, et al. A randomized controlled trial to assess the incidence of new onset hypertension in patients after shock wave lithotripsy for asymptomatic renal calculi. J Urol 1998;160:1241-3.

30. Lingeman JE, Kulb TB. Hypertension following extracorporeal shock-wave lithotripsy. J Urol 1987;137:142A.

31. Janetschek G, Frauscher F, Knapp R, Hofle G, Peschel R, Bartsch G. New onset hypertension after extracorporeal shockwave lithotripsy: age related incidence and prediction by intrarenal resistive index. J Urol 1997;158:346-51.

32. Zanetti G, Ostini F, Montanari E, Russo R, Elena A, Trinchieri A, et al. Cardiac dysrhythmias induced by extracorporeal shockwave lithotripsy. J Endourol 1999;13:409-12.

33. Asroff SW, Kingston TE, Stein BS. Extracorporeal shock wave lithotripsy in patient with cardiac pacemaker in an abdominal location: case report and review of the literature. J Endourol 1993;7:189-92.

34. Deliveliotis C, Kostakopoulos A, Stavropoulos N, Karagiotis E, Kyriazis P, Dimopoulos C. Extracorporeal shock wave lithotripsy in 5 patients with aortic aneurysm. J Urol 1995;154:1671-2.

35. Al Karawi MA, Mohamed AR, el-Etaibi KE, Abomelha MS, Seed RF. Extracorporeal shock-wave lithotripsy (ESWL)-induced erosions in upper gastrointestinal tract. Prospective study in 40 patients. Urology 1987;30:224-7.

36. Ohmori K, Matsuda T, Horii Y, Yoshida O. Effects of shock waves on the mouse fetus. J Urol 1994;151:255-8. 